\title{
Activated ceramic materials with deposition of photocatalytic titano-silicate micro-crystals
}

\author{
P. De Luca ${ }^{1}$, A. Chiodo ${ }^{1} \&$ J. B. Nagy ${ }^{2}$ \\ ${ }^{I}$ Department of Planning, University of Calabria, Italy \\ ${ }^{2}$ Department of Chemical and Materials Engineering, \\ University of Calabria, Italy
}

\begin{abstract}
The aim of this work was to prepare materials for use in buildings depollution, formed by titanosilicate microcrystals immobilized on ceramic surfaces, combining their unique properties, including the photo-catalytic properties of titanosilicates with high mechanical strength, with the thermal and chemical stability of the ceramic materials. In particular, titanosilicates, ETAS-10, belonging to the family of ETS (Engelhard titanium silicate), were studied.

Keywords: titanosilicate, ETAS-10, ETS-10, ceramic material, ceramic support, microcrystal, zeolite, multifunctional material, hydrothermal reaction, photocatalytic material.
\end{abstract}

\section{Introduction}

Today's environmental problems are forcing the search to find new materials with low environmental impact capable of responding to the increasingly looming ecological requirements. Through the development of knowledge and technology, new materials used in building and construction have undergone a considerable evolution in response to new standards of quality and possessing new and more articulate properties [1].

The application of new techniques to ceramic materials is a broad experimental field and involves a wide range of possible applications and uses so that, through coupling them with other materials, such as zeolites, new characteristics to the traditional properties of ceramic materials can be added.

The various applications of zeolites derive directly from their unique and diverse properties as ion exchange, catalysis, adsorption, molecular sieving [2], 
but in addition to these, in recent years, their photocatalytic properties were increasingly taken into account, in the same way as for traditional photocalytic agents such as, for example, titanium oxide [3].

Among the improvements sought in recent years in zeolite photochemistry, new zeotype materials, with porous nature, called ETS (Engelhard Titanium Silicate) [4-6], are included due to their structural properties to make them functional on photocatalytic processes and then with potential applicability in the degradation of pollutants.

These titanosilicates microporous materials have recently attracted attention as photocatalysts for two particular aspects: the first is that the ETS are semiconductors containing photo-excited titanium fractions, the second is that their structure is characterized by a series of three-dimensional channels $[7,8]$. The first aspect is certainly the most important in terms of active photocatalytic process for the formation of photo-induced torque (electron and hole), responsible for initiation of redox processes that lead to the degradation of pollutants. The second aspect, however, allows the titanosilicates to act as photoselector form with respect to the pollutants molecules with which it comes into contact.

In order to modify the properties, aluminum has been incorporated into the structure of ETS-10 phase giving rise to a new type of microporous material called titanium-aluminum-silicate ETAS-10 [9].

Many researchers have studied the synthesis of ETAS-10 with different contents of aluminum in the structure. They have shown that the structure of this material is closely similar to that of ETS-10. The ETAS-10 features all the properties and application capabilities of the ETS-10 as catalysis, adsorption, ion exchange and photo-reactivity [10].

In this work the experimental conditions were sought to optimize the deposition of micro crystals of ETAS-10 phase on different ceramic types supports.

The ultimate aim was to investigate the possibility of creating de-polluting material usable for buildings, made of micro crystals immobilized on ceramic surfaces, which can combine the unique properties of zeolites, including those photo-catalytic ones, with the high mechanical strength, thermal and chemical stability of ceramic materials.

\section{Experimental part}

The experimentation was divided into distinct but subsequent events.

\subsection{Preparation of different types of ceramic supports}

In particular, different support types were prepared: (a) only clay, (b) clay and $\mathrm{TiO}_{2}$ (Tioxide anatase), (c) clay and dried gel precursor of ETAS-10 phase (d) clay and crystalline ETAS-10. All ceramic supports were prepared using the following system: 
100g. Clay : 35g. Distilled water : 10g. Additional phase

where additional phase=titanium oxide; dry gel precursor of the ETAS-10 phase; ETAS- 10 crystalline phase.

The clay was pulverized by a pulverizer prior Fritsch Pulverisette model 2102 and then dried in an oven at $100^{\circ} \mathrm{C}$ for 1 hour. Titanium oxide, $\mathrm{TiO}_{2}$, as commercial product (anatase, Alfa Aesar) was used. The dry gel and the crystalline phase were prepared using the following synthesis system expressed in moles:

$$
1 \mathrm{Na}_{2} \mathrm{O}: 0.6 \mathrm{KF}: 0.2 \mathrm{TiO}_{2}: 1.28 \mathrm{HCl}: 1.49 \mathrm{SiO}_{2}: 0.03 \mathrm{Al}_{2} \mathrm{O}_{3}: 39.5 \mathrm{H}_{2} \mathrm{O}
$$

In particular, were prepared two solutions, one acidic and one basic. The acid solution was prepared by mixing $\mathrm{HCl}$ (Carlo Erba, $37 \mathrm{wt} \%$ ), $\mathrm{TiCl}_{4}$ (Carlo Erba, $50 \mathrm{wt} \%), \mathrm{AlCl}_{3} * 6 \mathrm{H}_{2} \mathrm{O}$ ( Merck) and distilled water. The basic solution was prepared by mixing $\mathrm{KF}$ (Merck, $40 \mathrm{wt} \%$ ), $\mathrm{SiO}_{2}$ (Merck) and $\mathrm{NaOH}$ solution (BDH Analar, 50wt\%). The two solutions were then mixed and a gel was obtained. Part of this gel was dried in an oven for 24 hours at a temperature of about $100^{\circ} \mathrm{C}$ and then pulverized, thus obtaining the powder of ETAS-10 dry gel, non-crystalline, to be included later in the support. The other part of the gel was poured into a cylindrical teflon, which in turn was inserted into an autoclave of stainless steel type Morey. Then everything was placed in an oven at $190{ }^{\circ} \mathrm{C}$ for 72 hours after which the autoclaves were cooled under running water for about 15 minutes.
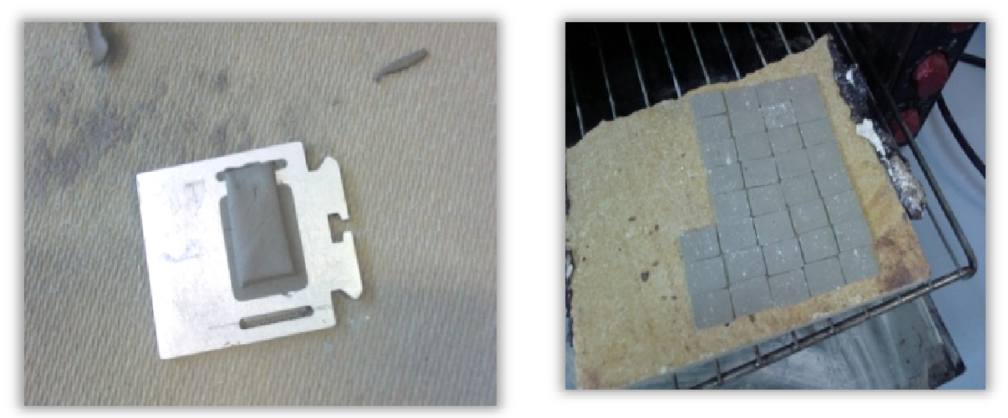

Figure 1: $\quad$ Shaping of ceramic supports in the slide for the X-ray diffraction.

The so prepared initial mixture, in order to achieve an adequate plasticity, was molded in the form of X-ray diffraction slides to be easily analyzed by XRD diffraction, fig. 1 .

The supports have been previously dried at $100^{\circ} \mathrm{C}$ for 24 hours and subsequently cooked in the oven at $600^{\circ} \mathrm{C}$ for two hours. The product was recovered by filtration and characterized by X-ray diffraction to ascertain the true nature of the phase and its crystallinity. The spectra obtained from XRD showed that the phase is ETAS-10 as it can be seen in fig. 2 . 


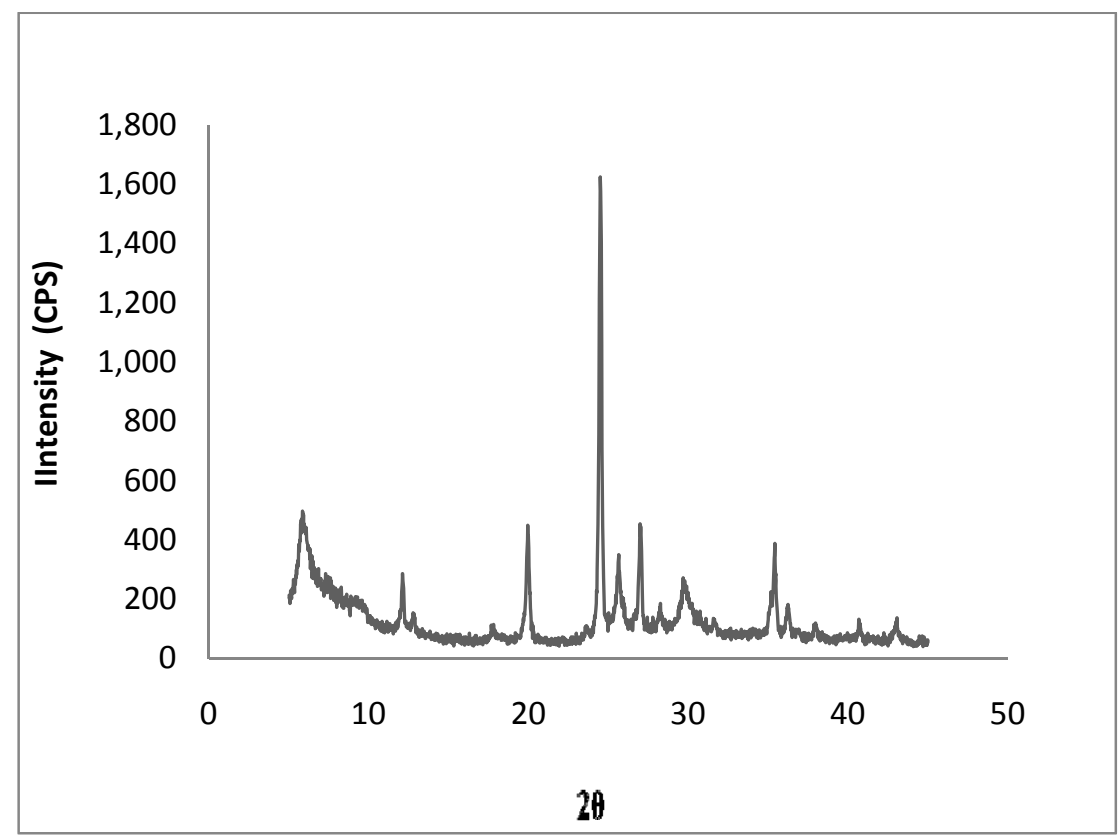

Figure 2: XRD patterns of ETAS-10 obtained at reaction temperature of $190^{\circ} \mathrm{C}$ and reaction time of 72 hours.

\subsection{Activation of the surface of the ceramic supports}

A part of the four different types of ceramic supports were subjected to a surface treatment, through a basic attack using a solution of 50 weight $\%$ of $\mathrm{NaOH}$ and leaving the supports in contact with it for 48 hours.

At the end a total of eight different supports were then obtained that have helped search for the best experimental conditions to obtain an optimal anchorage of the micro crystals on the surface of the supports as a function of the nature of added component and of the surface treatment.

\subsection{Deposition of micro-crystals titanium silicate ETAS-10 through one or more cycles of hydrothermal reaction}

The deposition of micro-crystals on the surface of the support was carried out through hydrothermal synthesis. In particular, each support was put in the precursor gel of ETAS-10 phase prepared as explained in Section 2.1, and the whole system was inserted in Morey type autoclaves and treated at a temperature of $190^{\circ} \mathrm{C}$ for 72 hours. After the expiry of the predetermined time, the autoclaves were cooled and then opened. The final products were thus: the support, a solution and micro-crystals deposited on the bottom of autoclaves, fig. 3. The support was then removed and washed with distilled water and dried at $100^{\circ} \mathrm{C}$ for 24 hours. The rest was filtered to recover the micro-crystals powder, and also 
they were washed and dried at $100^{\circ} \mathrm{C}$ for 24 hours. Subsequent XRD diffraction analysis was carried out both on the support and on the micro-crystal powder in order to verify that the phase obtained by initial gel was the same as that deposited on the support. On the same support were carried out several cycles of hydrothermal reaction in order to improve the deposition.
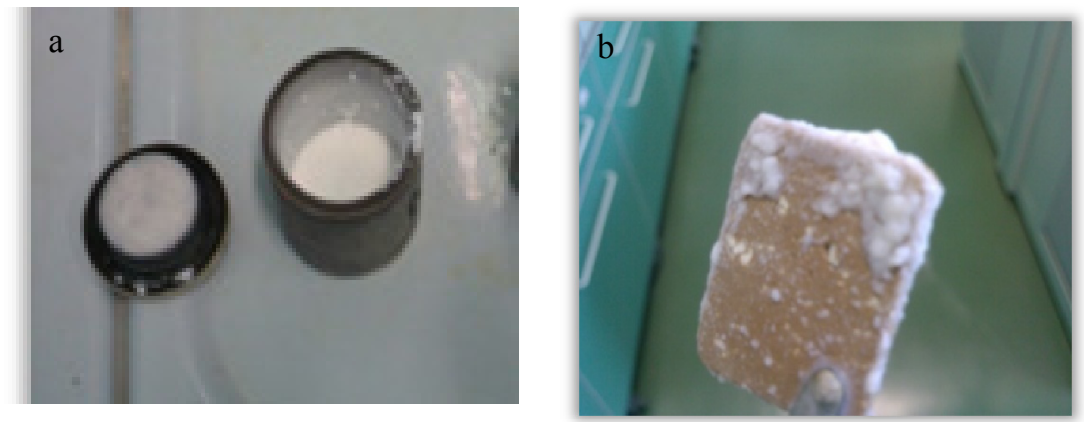

Figure 3: (a)Teflon container containing the final products. (b)Support extract before being washed.

\subsection{Chemical-physical characterization of obtained samples}

All samples were characterized by X-ray diffraction analysis (model Philips PW 1830) with $\mathrm{Cu} \mathrm{K} \alpha$ radiation, the scanning speed was $0.02^{\circ}$ per second and a range of between 5 and 452 Theta.

In order to allow a comparison between the crystallinity of various samples, the XRD spectrum of ETAS-10 phase powder obtained after 72 hours of reaction at $190{ }^{\circ} \mathrm{C}$ was taken as reference. To do this, two characteristic peaks, in particular, those at 20.05 and 24.57 2Theta and their respective relative and peak intensities were taken as a reference, fig.2. Applying the following expression:

$$
\text { Crystallinity }=\left[\left(\mathrm{I}_{\text {peak }}\right)_{1 *} *\left(\mathrm{I}_{\text {rel }}\right)_{1}+\left(\mathrm{I}_{\text {peak }}\right)_{2 *}\left(\mathrm{I}_{\text {rel }}\right)_{2}\right] / 2
$$

a value of crystallinity was obtained which has been assigned a value of 100 . Using the same procedure, the percentages were calculated for all crystalline samples. Scanning electron microscopy SEM Stereoscan (360S) was used to determine the best conditions for the crystals growth on the ceramic surface, depending on the support type, the number of hydrothermal reaction cycles and surface pretreatments. Moreover, the compressive strength of the samples was measured to study the influence of hydrothermal treatments on the mechanical properties of the ceramic support by hardness tester Vander Kamp VK200.

\section{Results and discussion}

All samples obtained showed a change in visual appearance of the surface as a function of the type of support, of the repeated cycles of reaction and of the surface treatment, fig. 4. 


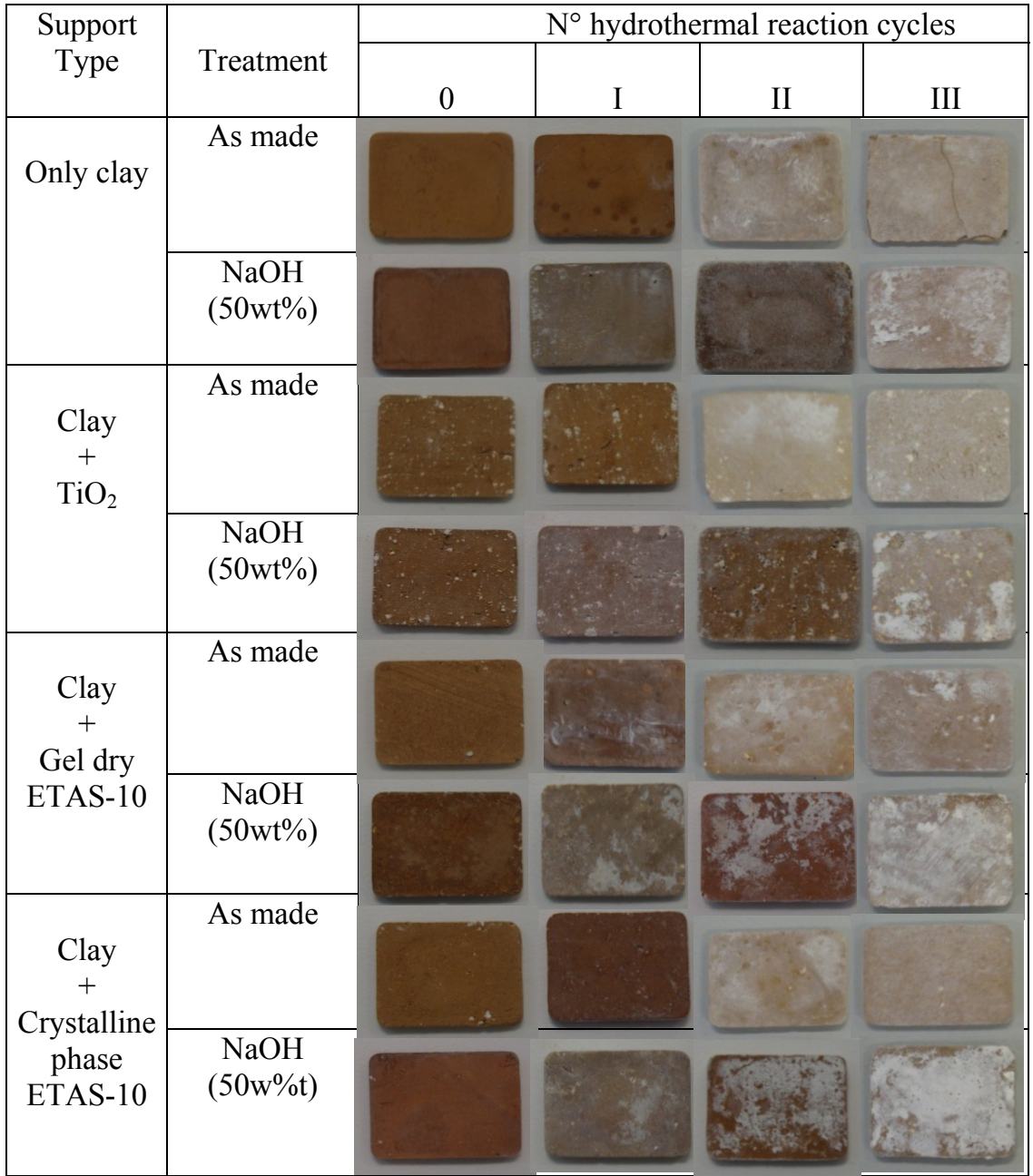

Figure 4: Samples obtained after repeated cycles of reaction depending on the type of substrate or surface treatment.

Images in fig. 4 show that the samples undergo, from first cycle to third cycle, a color change that becomes more evident in untreated support. The lighter color is logically independent of the firing temperature of the ceramic, since they are all cooked initially at the same temperature but probably this is due to reactions that occur during the hydrothermal reaction cycles, where the reaction gel has a basic $\mathrm{pH}$ around 12 .

The results obtained by X-ray diffraction analysis were analyzed to understand the role played by the nature of the support, the number of cycles of hydrothermal reaction and surface treatment on the deposition of crystals of ETAS-10 phase. 
The following figures 5-7 summarize a comparison of all experimental variables considered.

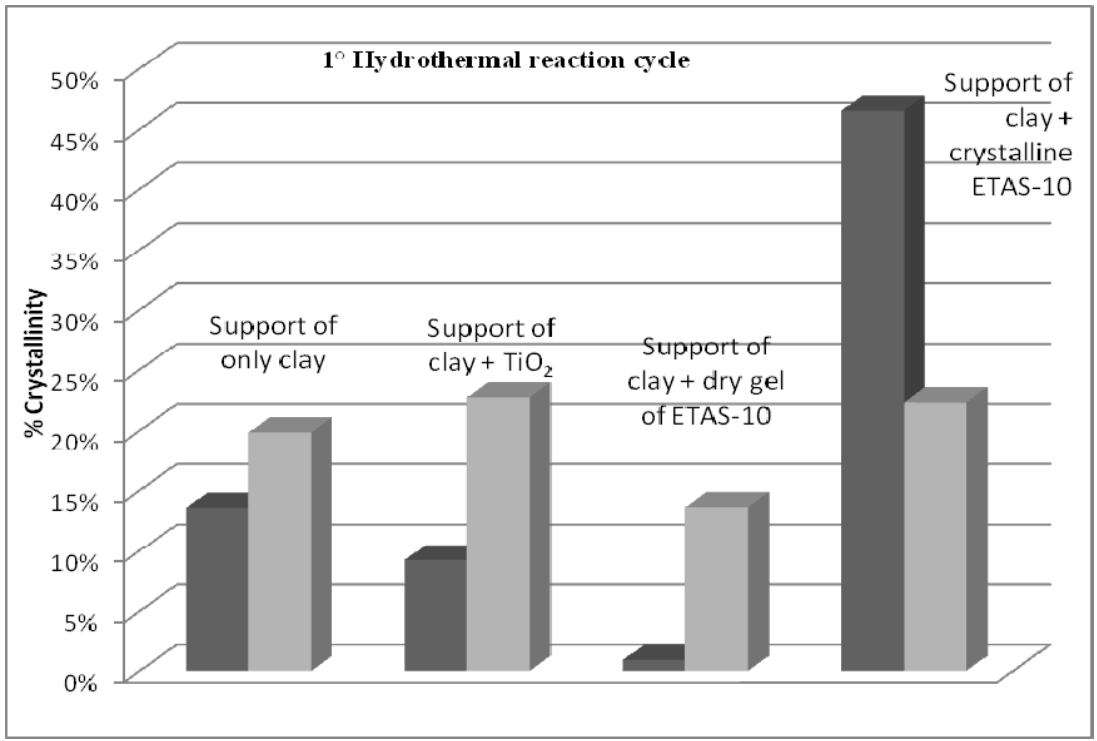

Figure 5: Crystallinity of samples obtained with one cycle of hydrothermal reaction as a function of support type and superficial treatment Untreated; Treated with $\mathrm{NaOH}$.

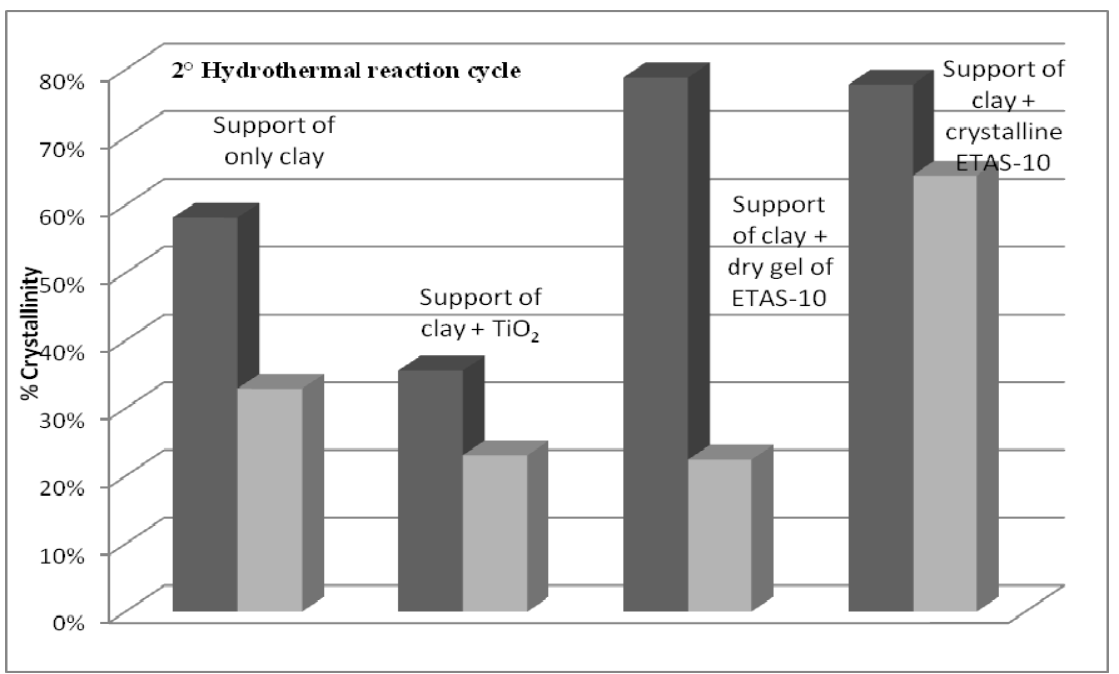

Figure 6: Crystallinity of samples obtained with two cycles of hydrothermal reaction as a function of support type and superficial treatment Untreated; Treated with $\mathrm{NaOH}$. 


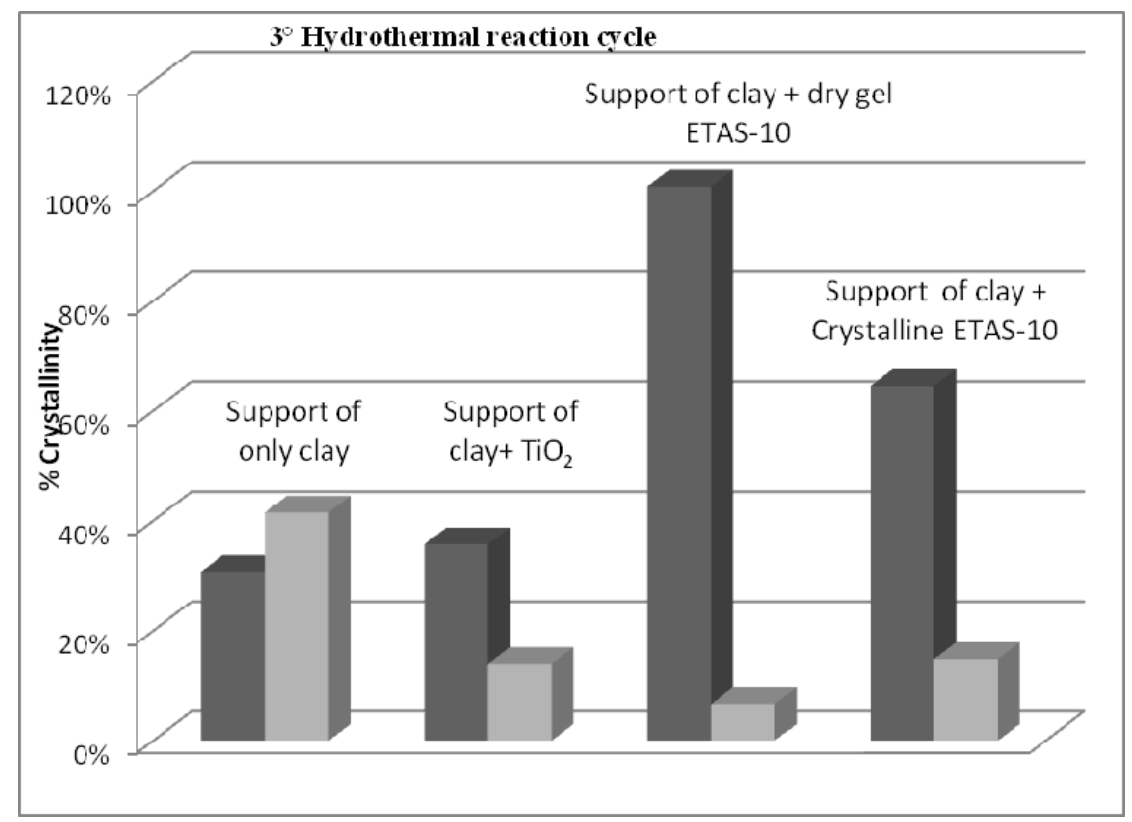

Figure 7: Crystallinity of samples obtained with three cycles of hydrothermal reaction as a function of support type and superficial treatment Untreated; Treated with $\mathrm{NaOH}$.

Considering the preceding figures, the crystallinity of the different samples obtained, it is possible to make some observations.

Pretreatment with $\mathrm{NaOH}$ solution, improves the deposition only in the first reaction cycle if compared to that obtained on support that did not undergo pretreatment.

With the succession of reaction cycles, the basic activation of the substrate surface becomes less influential. This can be justified because the initial gel has an alkaline $\mathrm{pH}$ around 12 and therefore from the second reaction cycle all supports suffer the same basic attack. The type of support plays a key role.

It was possible to observe that the best crystalline deposition takes place on support prepared from clay and dry gel precursor phase ETAS-10. This is easily explained by the fact that the dry gel inserted into the ceramic substrate is able to provide the nutrients necessary for the formation of the first crystals in the right molar ratios. To this can be added the peculiarity that the gel is not only deposited on the support but it is an integral part thereof and thus it is capable of providing a potential accession to the initial growth of crystals.

The number of cycles affects the growth of micro-crystals on the support.

It was possible to observe that, for almost all samples, in the first cycle of reaction, the crystallinity, as evidenced by X-ray diffraction, remains fairly low, which indicates a low surface coverage. There are still obvious XRD peaks attributable to the support. 
The second reaction cycle has an apparent lowering of the peaks (XRD) intensity of the support and an increase of peaks (XRD) due to the ETAS-10 phase thus confirming a good coverage of the substrate surface.

At the third reaction cycle there is a decrease of crystallinity but in some cases, only slight lowering occurs.

This could be justified by assuming a "saturation" of the surface of the support sites that are not able to trigger the growth of new crystallization nuclei and also to a partial detachment of micro-crystals, which were formed in previous cycles.

The best result was obtained on the support containing dry gel precursor of ETAS-10 phase, without pre-treatment with $\mathrm{NaOH}$, and at the third cycle of synthesis.

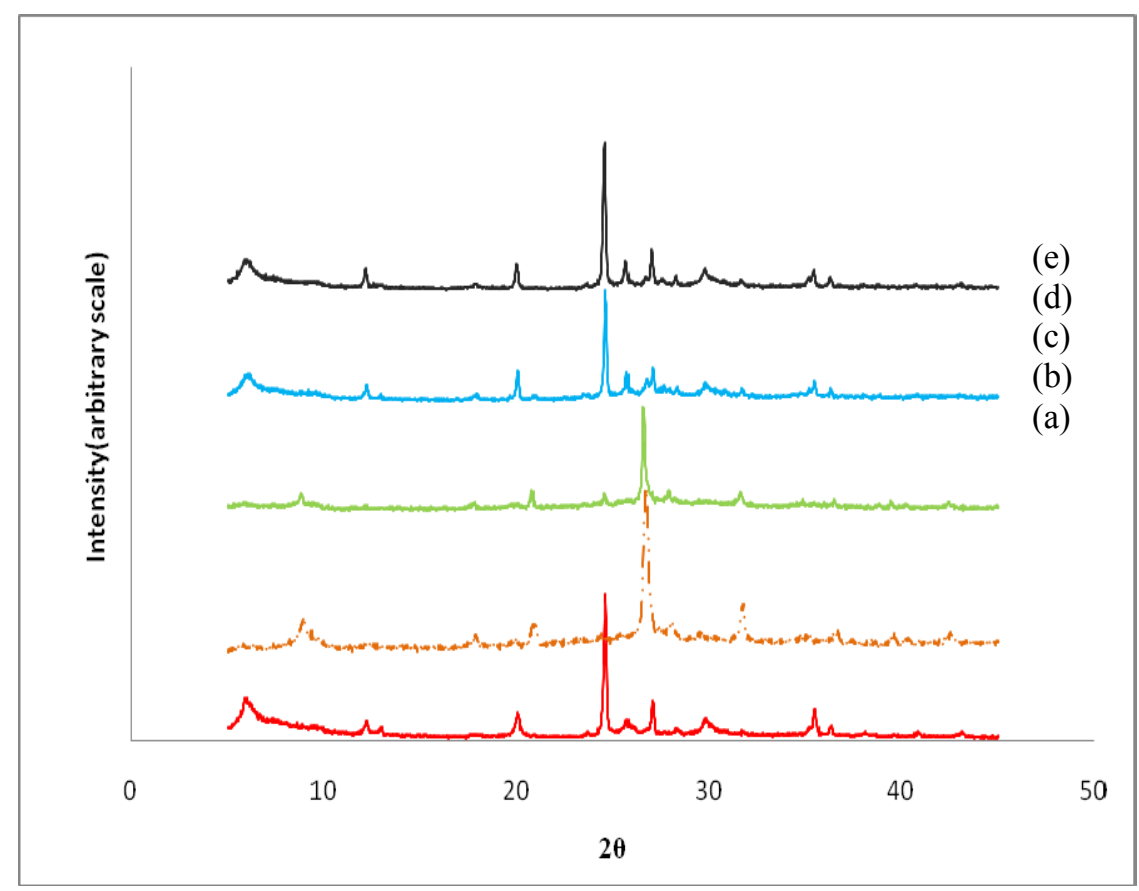

Figure 8: Comparison between XRD spectra of: (a) ETAS-10 powder; (b) support with clay and dry gel of ETAS-10 as made; (c) at first cycle; (d) at 2 nd cycle; (e) at 3 rd cycle.

The mechanical properties on all samples obtained were performed to verify if the different treatments, basic attack and hydrothermal reaction, affect their characteristics.

In the case of the treated support this threshold was consistently exceeded, although some effect of cleavage appeared on the supports treated and to the third cycle, revealing a strengthening of the support in contact with the $\mathrm{NaOH}$ solution. 
Table 1: Compressive strength values of $\mathrm{Kp}$ (Kilopound) of the various supports. $\mathrm{UT}=$ untreated, $\mathrm{T}=$ treated with $\mathrm{NaOH}$.

\begin{tabular}{|c|c|c|c|c|c|c|c|c|}
\hline & \multicolumn{2}{|c|}{$\begin{array}{c}\text { Support } \\
\text { Only clay }\end{array}$} & \multicolumn{2}{|c|}{$\begin{array}{c}\text { Support of } \\
\text { Clay }+\mathrm{TiO}_{2}\end{array}$} & \multicolumn{2}{|c|}{$\begin{array}{c}\text { Support of } \\
\text { Clay + dry gel of } \\
\text { ETAS-10 }\end{array}$} & \multicolumn{2}{c|}{$\begin{array}{c}\text { Support of } \\
\text { Clay }+ \\
\text { crystalline } \\
\text { ETAS-10 }\end{array}$} \\
\cline { 2 - 10 } & $\mathrm{UT}$ & $\mathrm{T}$ & $\mathrm{UT}$ & $\mathrm{T}$ & $\mathrm{UT}$ & $\mathrm{T}$ & $\mathrm{UT}$ & $\mathrm{T}$ \\
\hline As made & 20.6 & $>35$ & 15.9 & $>35$ & $>35$ & $>35$ & 22.0 & $>35$ \\
\hline 1 cycle & 22.0 & $>35$ & 17.5 & $>35$ & $>35$ & $>35$ & $>35$ & $>35$ \\
\hline 2 cycle & 20.6 & $>35$ & $>35$ & $>35$ & $>35$ & $>35$ & $>35$ & $>35$ \\
\hline 3 cycle & 25.2 & $>35$ & 32.9 & $>35$ & $>35$ & $>35$ & $>35$ & $>35$ \\
\hline
\end{tabular}
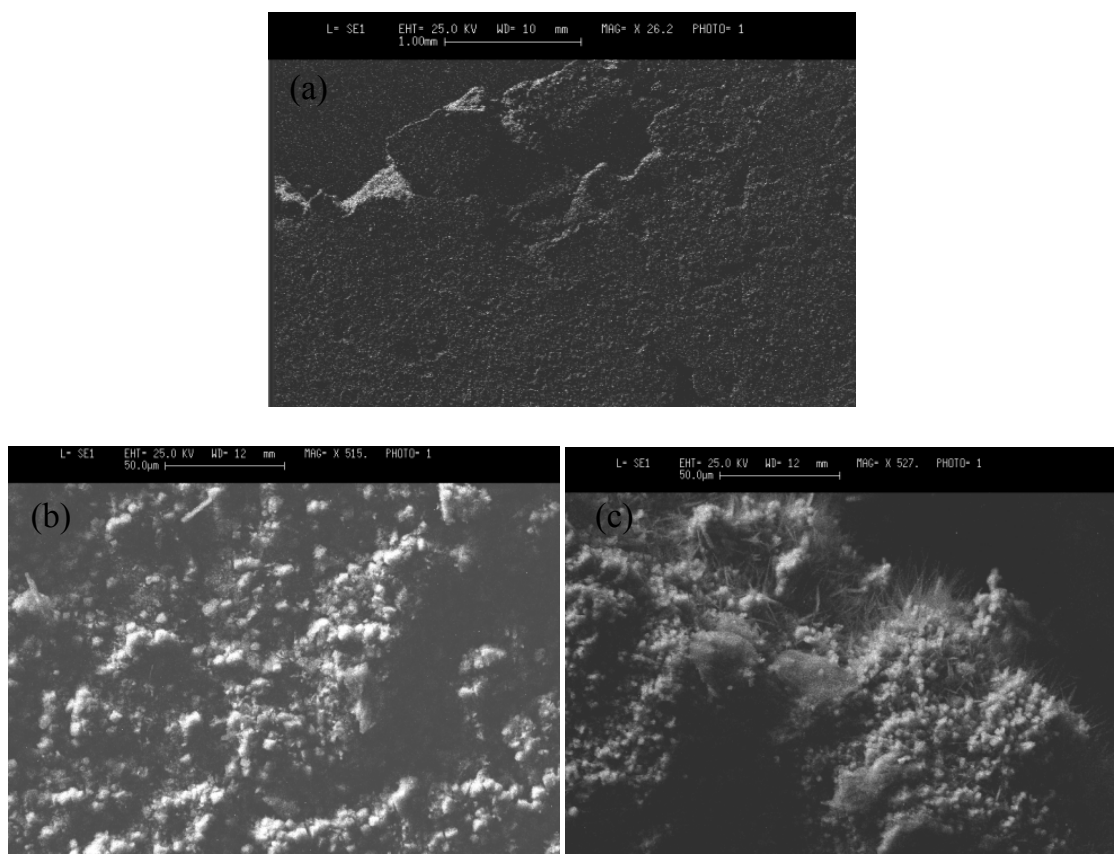

Figure 9: SEM micrographs of the support containing dry gel phase ETAS10: (a) as made, (b) after 1 cycle of hydrothermal reaction (c) after the 2 nd cycle of hydrothermal reaction.

The instrument used is calibrated to values not exceeding $35 \mathrm{Kp}$ (kilopounds).

The same phenomenon happened on untreated support increasing the number of cycle so it could be inferred that, in general, as regards to mechanical resistance to compression, supports activated with $\mathrm{NaOH}$ showed the best results. 
The SEM investigation has validated the data obtained through the diffractometric XRD study, highlighting the progressive growth of crystals with increasing hydrothermal treatment and a good anchorage of the crystals on the surface of the substrate.

\section{Conclusion}

The combined results obtained allow the following conclusions. The best experimental conditions for the deposition of micro-crystals of ETAS-10 phase on ceramic supports were obtained on support prepared with dry gel precursor phase ETAS-10 in the second reaction cycle hydrothermal without pre-basic treatment.

\section{References}

[1] Benedix, R., Dehn, F., Quaas, J., Orgass, M., Application of Titanium Dioxide Photocatalysis to Create Self-Cleaning Building Materials, Lacer, 5, pp. 157-166, 2000.

[2] B. Nagy, J., Bodart, P., Hannus, I., Kiricsi, I., Synthesis, characterization and use of zeolite microporous materials, DecagenLtd, Szeged, Hungary, 1998.

[3] Dutta, P.K., Kim Y., Photochemical process in zeolites: new developments, pp. 483-489, 2004.

[4] Kuznicki, S.M., U.S. Pat. 4, 853,202 (1989).

[5] Kuznicki, S.M., Thrush, A.K., Eur. Pat. 0405978A1 (1990).

[6] Pavel, C.C., Vuono, D., Catanzaro, L., De Luca, P., Bilba, N., Nastro, A., B.Nagy, J., Synthesis and characterization of the microporous titanosilicates ETS-4 and ETS-10. Microporous and Mesoporous Materials, 56, pp. 227-239, 2002.

[7] Shough, A.M., Doren, D.J., Nash, M., Lobo, R.F., Effect of Vanadium Substitution on the Structure and Photocatalytic Behaviour of ETS-10, J. Phys. Chem C., 111(4), pp.1176-1782, 2007.

[8] Guan, G., Kida, T., Kusacabe, K., Kimura, K., Abe, E., Yoshude, A., Photocatalytic reactivity of noble metal-loaded ETS-4 zeolites, Chem. Comm., 7, pp. 618-620, 2004.

[9] Calza, P., Pazè, C., Pellizzetti, E., Zecchina, A., Shape-selective photocatalytic transformation of phenols in an aqueous medium. Chemical Communication, pp. 2130-2131, 2001.

[10] Liepold, A., Roos, K., Reschetilowski, W., Lin, Z., Rocha, J., Philippou , A., Anderson, M. W., Characterisation studies on the new microporous aluminiumcontaining ETS-10 molecular sieve used for processing larger molecules. Microporous Materials, 10, pp. 211-224, 1997.

[11] Kim, S. D., Noh, S. H., Kim, Y. C., Hwang, J. Y., Jung, J. Y., Yi Sung Churl, Ki, W. J., Hydrothermal synthesis of aluminum-substituted titanosilicate, ETS-10, J. Porous Materials, 16, pp. 307-314, 1997. 